L. Ein and I. Sols

Nagoya Math. J.

Vol. 96 (1984), 11-22

\title{
STABLE VECTOR BUNDLES ON QUADRIC HYPERSURFACES
}

\author{
L. EIN AND I. SOLS
}

$\S 0$.

Barth, Hulek and Maruyama have showed that the moduli of stable rank 2 vector bundles on $\boldsymbol{P}^{2}$ are nonsingular rational varieties. There are also many examples of stable rank 2 vector bundles on $P^{3}$. On the other hand, there is essentially only one example of rank 2 bundles on $P^{4}$, which is constructed by Horrocks and Mumford. We hope the study of rank 2 bundles on hypersurfaces in $P^{4}$ may give more insight to the study of vector bundles on $\boldsymbol{P}^{4}$. In this paper, we establish some general properties of stable rank 2 bundles on quadric hypersurfaces. We show the restriction theorem (1.4), (1.6), the existence of the spectrum (2.2), and the vanishing theorem (2.4), are also true for the stable rank 2 reflexive sheaves on quadric hypersurfaces just as in the case when the base variety is $\boldsymbol{P}^{n}$. Though the methods to prove such results are similar to those we use for projective spaces, there are some technical difficulties. We should also mention that we shall always assume the base field is characteristic 0 and algebraically closed, and we shall use the definition of stability introduced by Mumford and Takemoto.

$\S 1$.

We let $Q_{n}$ be a nonsingular quadric hypersurface in $\boldsymbol{P}^{n+1}$. There is the following incidence correspondence:

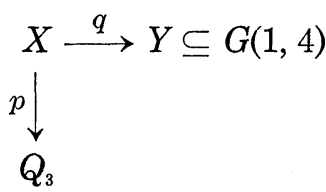

where $Y$ is the subvariety in $G(1,4)$, which corresponds to the set of lines in $Q_{3} . \quad X$ is the corresponding universal $P^{1}$-bundle on $Y$. It is not hard to check that $X$ a conic bundle over $Q_{3}$.

Received May 9, 1983. 
Lemma 1.1. Let $x \in Y$ and $L=q^{-1}(x)$. Then

$$
\left.\Omega_{X / Q_{3}}^{1}\right|_{L} \cong \mathcal{O}_{P 1}(1) \text {. }
$$

Proof. There are the following exact sequences:

$$
0 \longrightarrow I_{L} /\left.I_{L}^{2} \longrightarrow \Omega_{X / k}^{1}\right|_{L} \longrightarrow \Omega_{L / k}^{1} \longrightarrow 0
$$

and

$$
\left.\left.\left.0 \longrightarrow p^{*} \Omega_{Q_{3} / k}^{1}\right|_{L} \longrightarrow \Omega_{X / k}^{1}\right|_{L} \longrightarrow \Omega_{X / Q_{3}}^{1}\right|_{L} \longrightarrow 0 \text {. }
$$

Because $I_{L} / I_{L}^{2} \cong 3 \mathcal{O}_{L}$ and $\left.\Lambda^{3} \Omega^{1} Q_{3 / k}\right|_{L} \cong \mathcal{O}_{P 1}(-3)$, it is easy to show $\left.\Omega_{X / Q_{3}}^{1}\right|_{L}$ $\cong \mathcal{O}_{L}(1)$ by Chern classes computation.

Definiton 1.2 (Mumford-Takemoto). A reflexive sheaf $E$ of rank $r$ on $Q_{n}$ is stable (respectively, semistable) if for every proper subsheaf $F$ $(1 \leq \operatorname{rank} F \leq \operatorname{rank} E-1)$,

$$
\frac{c_{1}(F) \cdot c_{1}\left(\mathcal{O}_{Q_{n}}(1)\right)^{n-1}}{\operatorname{rank} F}<\frac{c_{1}(E) \cdot c_{1}\left(\mathcal{O}_{Q_{n}}(1)\right)^{n-1}}{\operatorname{rank} E}
$$

(respectively, $\leq$ ).

Proposition 1.3. Let $E$ be a semistable reflexive sheaf of rank $r$ on $Q_{3}$. Suppose that $E_{L}$, the restriction of $E$ to a general line $L$, is isomorphic to $\oplus_{j=1}^{r} \mathcal{O}_{p_{1}}\left(a_{j}\right)$ with $a_{1} \leq a_{2} \leq \cdots \leq a_{r}$. Then $a_{i+1}-a_{i} \leq 1(1 \leq i \leq r-1)$.

Proof. The proof is fairly standard ([6]) and we shall merely give a sketch. We shall use the notations in the diagram 1.A. Suppose $a_{i+1}-$ $a_{i}>1$. It is easy to construct a sheaf $F \subseteq p^{*} E$ of rank $i$ such that $\left.F\right|_{L} \cong \oplus_{j=1}^{i} \mathcal{O}\left(a_{j}\right)$ and $p^{*} E /\left.F\right|_{L}=\left.G\right|_{L} \cong \bigoplus_{j=r+1}^{r} \mathcal{O}\left(a_{j}\right)$.

Let $U \subseteq Q_{3}$ be the open set where $E$ is locally free. There is a set $V \subseteq X$ such that $\left.F\right|_{V}$ and $\left.G\right|_{V}$ are locally free. By the universal property of $\operatorname{Gr}\left(E_{U}\right)$, there is a morphism $f: V \rightarrow G r\left(E_{U}\right)$

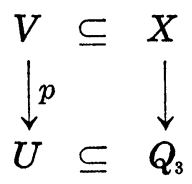

such that $G$ is the pull back of the universal quotient bundle. Since $\left.\left.f^{*} \Omega_{G r / U}^{1}\right|_{L} \cong F \otimes G^{*}\right|_{L}$ and $a_{i+1}-a_{i}>1$,

$$
d f:\left.\left.f^{*} \Omega_{G r / U}^{1}\right|_{L} \longrightarrow \Omega_{v / U}^{1}\right|_{L} \cong \mathcal{O}_{L}(1)
$$


(Lemma 1.1) is the zero map. So $d f=0$ therefore $f$ factors through $p$ and $E$ will have a subsheaf $F^{\prime}$, with the property $\left.F^{\prime}\right|_{L} \cong \oplus_{j=1}^{i} \mathcal{O}_{L}\left(a_{j}\right)$, which contradicts the fact that $E$ is semistable.

Proposition 1.4. Let $E$ be a semistable vector bundle of rank $r$ on $Q_{2}$. Suppose $E_{H}$, the restriction of $E$ to a general hyperplane section (conic), is isomorphic to $\oplus_{i=1}^{r} \mathcal{O}_{P_{1}}\left(a_{i}\right)$ with $a_{1} \leq a_{2} \leq \cdots \leq a_{r}$. Then $a_{i+1}-a_{i} \leq 1$.

Proof. The proof is similar to the proof of Proposition 1.3. We shall leave it to the readers.

CoRollaRy 1.5. Let $E$ be rank 2 semistable rank 2 vector bundle on $Q_{2}$, such that $\Lambda^{2} E \cong \mathcal{O}_{Q_{2}}$ (respectively, $\left.\Lambda^{2} E \cong \mathcal{O}_{Q}(-1)\right)$. Then $E_{H}$, the restriction of $E$ to a general hyperplane section (conic), is isomorphic to $\mathcal{O}_{P^{1}} \oplus \mathcal{O}_{P^{1}}$ (respectively, $\mathcal{O}_{\boldsymbol{P} 1}(-1) \oplus \mathcal{O}_{\boldsymbol{P} 1}(-1)$ ).

Let $\left[Q_{2}\right],[L]$, and $[P]$ (quadric surface, line, and point) be the generators of $H^{2}\left(Q_{3}, Z\right), H^{4}\left(Q_{3}, Z\right)$, and $H^{6}\left(Q_{3}, Z\right)$ respectively. There are the following relationships: $\left[Q_{2}\right]^{2}=2[L]$ and $\left[Q_{2}\right] \cdot[L]=2[P]$. If $F$ is a coherent sheaf on $Q_{3}$, the Chern polynomial of $F$ is of the form $1+c_{1}(F)$ $\left[Q_{2}\right] t+c_{2}(F)[L] t^{2}+c_{3}(F)[P] t^{3}$. Often we shall simply call $c_{i}(F)=c_{i}$ the Chern classes of $F$.

It is not hard to show the following Riemann Roch formula:

$$
\chi(F)=\frac{1}{6}\left(2 c_{1}^{3}-3 c_{1} c_{2}+3 c_{3}\right)+\frac{3}{2}\left(c_{1}^{2}-c_{2}\right)+\frac{13}{6} c_{1}+\operatorname{rank}(F) .
$$

If $\operatorname{rank}(F)=2$, there are the following congruence relationships:

(a) $c_{1}=0$, then $c_{2}=c_{3}(\bmod 2)$

(b) $c_{1}=-1$, then $c_{3}=0(\bmod 2)$.

If $E$ is a rank 2 reflexive sheaf on $Q_{3}$, then as in [7] one can check that $c_{3}(E)=$ number of points where $E$ fails to be locally free.

THEOREM 1.6. Let $E$ be a rank 2 stable reflexive sheaf on $Q_{n}(n \geq 3)$. Then $E_{H}$, the restriction of $E$ to a general hyperplane, is again stable.

Proof. By tensoring $E$ by $\mathcal{O}_{Q_{n}}(t)$ if necessary, we may assume that $\Lambda^{2} E$ is either $\mathcal{O}_{Q_{n}}$ or $\mathcal{O}_{Q_{n}}(-1)$. We shall work out the details for the case $\Lambda^{2} E=\mathcal{O}_{Q_{n}}$. Since the proof of the other case is similar, we shall omit it.

We consider the following incidence correspondence. First we'll show $h^{0}\left(E_{H}\right)=0$. 


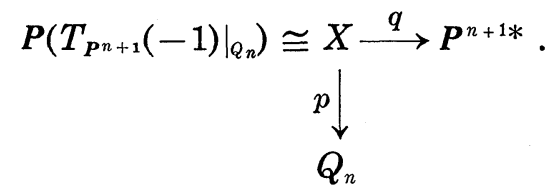

For $n \geq 4$, this will show that $E_{H}$ is stable. Let $a$ be the integer such that $h^{0}\left(E_{H}(a)\right) \neq 0$ and $h^{0}\left(E_{H}(a-1)\right)=0$. Suppose $a \leq 0$ and we shall derive a contradiction. Because $q_{*} p^{*} E(a) \neq 0$ and torsion free, there is the least integer $t$ such that $h^{0}\left(q_{*} p^{*} E(a) \otimes \mathcal{O}_{P^{n+1}}(t)\right) \neq 0$. We can construct the following exact sequence:

$$
0 \longrightarrow \mathcal{O}_{X}(-a,-t) \longrightarrow p^{*} E \longrightarrow I_{Z}(a, t) \longrightarrow 0 .
$$

(We are using the notation $\mathcal{O}_{X}(-a,-t)$ for $p^{*} \mathcal{O}_{Q_{n}}(-a) \otimes q^{*} \mathcal{O}_{P^{n+1 *}}(-t)$.) By the universal property of $\boldsymbol{P}_{Q_{n}}(E)$, there is a map $f: X-Z \rightarrow \boldsymbol{P}_{Q_{n}}(E)$ such that $f^{*} \mathcal{O}_{\boldsymbol{P}(E)}(1) \cong \mathcal{O}_{X-Z}(a, t)$ and $f^{*} \Omega_{\boldsymbol{P}(E) / Q_{n}}^{1} \cong \mathcal{O}_{X-Z}(-2 a,-2 t)$. According to Propositions 3.1 and 3.2 in [5], these are the following diagrams:

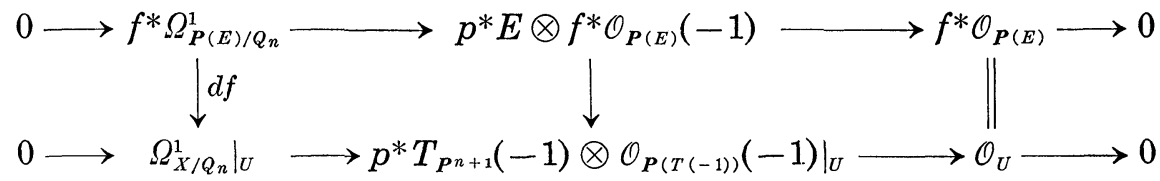

where $U=X-Z$. Also

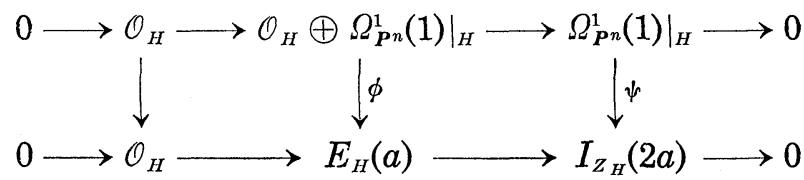

where rank $\psi \geq 1$ and $Z_{H}=Z \cap H$. However, Hom $\left(\left.\Omega_{P^{n}}^{1}(1)\right|_{H}, I_{Z_{H}}(2 a)\right) \subseteq$ $\operatorname{Hom}\left(\left.\Omega_{P^{n}}^{1}(1)\right|_{H}, \mathcal{O}_{H}(2 a)\right)=H^{0}\left(\left.T_{P^{n}}(2 a-1)\right|_{H}\right)$. If $a<0$, then $H^{0}\left(\left.T_{P^{n}}(2 a-1)\right|_{H}\right)$ $=0$. So we may assume $a=0$. Since $H^{0}\left(T_{P^{n}}(-1)\right) \cong H^{0}\left(\left.T_{P^{n}}(-1)\right|_{H}\right)$, any section of $\left.T_{P^{n}}(-1)\right|_{H}$ is either nowhere vanishing or vanishing at one point. If $n \geq 4$ and $\operatorname{dim} Z_{H} \geq 1$, then $\psi=0$ and we have a contradiction. On the other hand, if $Z_{H}=\phi, E_{H} \cong 2 \mathcal{O}_{H}$. In this case, $H^{1}\left(E_{H}(m)\right)$ $=0$ for all $m$. Therefore $H^{1}(E(m))=0$ for all $m$. Since $H^{1}(E(-(1))=0$, the restriction map $\operatorname{Hom}\left(2 \mathcal{O}_{Q}, E\right) \rightarrow \operatorname{Hom}\left(2 \mathcal{O}_{H}, E_{H}\right)$ is surjective. Hence $H^{0}(E) \neq 0, E$ is not stable. The remaining case is when $n=3$ and $Z_{H}$ is a simple point. In this case, $c_{2}(E)=1$ and $h^{1}\left(E_{H}(m)\right)=0$ for $m \neq-1$. Therefore $H^{1}(E(m))=0$ for $m \leq-2$. From the exact sequence, $0 \rightarrow E(t)$ $\rightarrow E(t+1) \rightarrow E_{H}(t+1) \rightarrow 0$, we find $h^{1}(E(-1)) \leq 1$. Since $h^{0}(E)=0$ and 
$h^{0}\left(E_{H}\right)=1, \quad h^{1}(E(-1)) \geq 1$. Then $h^{1}(E(-1))=1$ and $h^{1}(E(m))=0$ for $m \geq 0$. Thus $H^{0}(E(1)) \rightarrow H^{0}\left(E_{H}(1)\right)$ is surjective. There is an exact sequence $H^{0}(E(1)) \otimes \mathcal{O}_{Q_{3}} \rightarrow E(1) \rightarrow F \rightarrow 0$ with $\operatorname{dim} \operatorname{supp} F \leq 0$. Let $C$ be the zero set of a general section of $E(1)$. Then $C$ has at most finite number of singularities. The extension, $0 \rightarrow \mathcal{O}_{Q_{3}} \rightarrow E(1) \rightarrow I_{C}(2) \rightarrow 0$, is given by an element in $\operatorname{Ext}^{1}\left(I_{C}(2), \mathcal{O}_{Q_{3}}\right) \cong \operatorname{Ext}^{2}\left(\mathcal{O}_{C}(2), \mathcal{O}_{Q_{3}}\right) \cong H^{0}\left(\omega_{C}(1)\right)$. Furthermore, $C$ is not contained in any hyperplane, $\operatorname{deg} C=3$, and $h^{1}\left(I_{C}\right)=1$. So $C$ has two connected components and one of the components is a line $L$. But $h^{0}\left(\omega_{L}(1)\right)=0$. This contradicts the fact $E$ is reflexive, hence it can only fail to be locally free at finite number of points. So far we have shown $h^{0}\left(E_{H}\right)=0$. It remains to show that $E_{H}$ is stable when $n=3$. By Proposition 1.3, we may assume the restriction of $E$ to a general line in either rulings is isomorphic to $\mathcal{O}_{P_{1}} \oplus \mathcal{O}_{P_{1}}$. If there is an injective map $\mathrm{g}: \mathcal{O}_{H}(a, b) \rightarrow E_{H}$ with $a+b \geq 0$ and $h^{0}\left(\mathcal{O}_{H}(a, b)\right)$ $=0$, then we may assume $a>0$ and $b<0$. Then the restricting $g$ to a general line in the second ruling, we get a nonzero map $\mathcal{O}_{P^{1}}(a) \rightarrow 2 \mathcal{O}_{P^{1}}$ which is a contradiction. So $E_{H}$ is stable.

\section{$\S 2$.}

Let $E$ be a rank 2 stable reflexive sheaf on $Q_{3}$ with $c_{1}(E)=0, c_{2}(E)$ $=c_{2}$. We shall show that there is a vector bundle $\mathscr{H}$ of rank $c_{2}$ on $\boldsymbol{P}^{1}$, which we shall call the spectrum of $E$, such that $h^{1}(E(m))=h^{0}(\mathscr{H}(m+1))$ for $m \leq-1$. This is analogous to the results of Barth, Elencwajg, and Hartshorne ([3] and [7]) about the spectrums of stable reflexive sheaves of rank 2 on $\boldsymbol{P}^{3}$. The methods to prove this result are similar to those given by Hartshorne in [7]. We shall need the following technical lemma.

Let $\mathcal{O}_{Q_{2}}(1,0)$ and $\mathcal{O}_{Q_{2}}(0,1)$ be the line bundles associated with the rulings. In general, we write $\mathcal{O}_{Q_{2}}(a, b)$ for $\mathcal{O}_{Q_{2}}(a, 0) \otimes \mathcal{O}_{Q_{2}}(0, b)$.

LeMma 2.1. Let $E$ be a rank 2 vector bundle on $Q_{2}$.

$$
M=\bigoplus_{m \in Z} H^{1}\left(\left.E \otimes \mathcal{O}_{P^{3}}(m)\right|_{Q_{2}}\right) \text {. }
$$

Suppose $N \subseteq M$ be a graded submodule. Let $n_{t}=\operatorname{dim} N_{t}$ where $N_{t}$ is the graded component of $N$ in degree $t$.

(1) (a) If $E$ is semistable and $\Lambda^{2} E \cong \mathcal{O}_{Q_{2}}$ (respectively, $\mathcal{O}_{Q_{2}}(-1,-1)$ ) then $n_{t} \leq n_{t+1}$ for $t \leq-2$ (respectively, -1 ).

(b) If $n_{t} \neq 0$, then $1+n_{t} \leq n_{t+1}$ for $t \leq-3$ (respectively, $t \leq-2$ ). 
(2) If $E$ is semistable and $\Lambda^{2} E \cong \mathcal{O}_{Q_{2}}$ (respectively, $\mathcal{O}_{Q_{3}}(-1,-1)$ ), and $n_{-1} \neq 0$ (respectively, $0<n_{-1}<h^{1}(E(-1))$ ), then $1+n_{-2} \leq n_{-1}$ (respectively, $\left.1+n_{-1} \leq n_{0}\right)$.

(3) Suppose $E$ is semistable and $\Lambda^{2} E \cong \mathcal{O}_{Q_{2}}$ (respectively, $\mathcal{O}_{Q_{2}}(-1,-1)$ ). If $n_{t_{0}} \neq 0$ and $1+n_{t_{0}}=n_{t_{0}+1}$ for some $t_{0} \leq-3$ (respectively, $t_{0} \leq-2$ ), then there is a nonzero element $s \in H^{0}\left(\mathcal{O}_{Q_{2}}(1,0)\right)$ or $H^{0}\left(\mathcal{O}_{Q_{2}}(0,1)\right)$ such that $x \cdot N_{m}$ $=0$ for $m \leq t_{0}+1$ where $x \in H^{0}\left(\mathcal{O}_{Q_{2}}(1,1)\right)$ and $s \mid x$. Furthermore, if $y \in$ $H^{0}\left(\mathcal{O}_{Q_{2}}(1,1)\right)$ is not divisible by $s$, then $N_{m} \stackrel{y}{\longrightarrow} N_{m+1}$ is injective for $m \leq t_{0}+1$.

Proof. (1) (a) Suppose $H$ is a general hyperplane section of $Q_{2}$ defined by an element $x \in H^{0}\left(\mathcal{O}_{Q_{2}}(1)\right.$ ), such that $E_{H} \cong \mathcal{O}_{P^{1}} \oplus \mathcal{O}_{P^{1}}$ (Corollary 1.5). There is the exact sequence $0 \rightarrow E(t) \rightarrow E(t+1) \rightarrow E_{H}(t+1) \rightarrow 0$. Since $h^{0}\left(E_{H}(t+1)\right)=0$ for $t \leq-2$, the map $N_{t} \stackrel{x}{\longrightarrow} N_{t+1}$ is injective and $n_{t} \leq n_{t+1}$.

(b) We shall prove (b) by induction on the length of $N$. If $n_{t+1}<$ $3+n_{t}$, $(t \leq-3)$ then by the bilinear map lemma ([7], Lemma 5.1), there is an element $x \in H^{0}\left(\mathcal{O}_{Q_{2}}(1)\right)$, such that $N_{t} \stackrel{x}{\longrightarrow} N_{t+1}$ is not injective. Let $C$ be the conic defined by $x$.

Because $N_{t} \stackrel{x}{\longrightarrow} N_{t+1}$ is not injective, $h^{0}\left(E_{C}(t+1)\right) \neq 0$. There is the exact sequence:

$$
\oplus_{j} H^{0}\left(E_{C}(j)\right) \stackrel{\delta}{\longrightarrow} \oplus_{j} H^{1}(E(j-1)) \stackrel{x}{\longrightarrow} \oplus H^{1}(E(j)) .
$$

Set $U=\operatorname{Im}(\delta) \cap N(-1)$ and $V=\operatorname{Im}\left(\left.x\right|_{N(-1)}\right)$. Then there are the following exact sequences: $0 \rightarrow U \rightarrow N(-1) \rightarrow V \rightarrow 0$ and $0 \rightarrow V \rightarrow N \rightarrow N / V \rightarrow 0$. We notice that $V$ is also a submodule of $N$. Furthermore, $U_{t}=\delta \delta^{-1}\left(N_{t}\right)$ for $t \leq 0$.

Case 1. Assume $C$ is nonsingular. Then $E_{C} \cong \mathcal{O}_{P_{1}}(a) \oplus \mathcal{O}_{P_{1}}(-a)$ with $2 t+2+a \geq 0$. Also it is easy to check that $\operatorname{dim} U_{j}+2 \leq \operatorname{dim} U_{j+1}$ if $U_{j} \neq 0$ and $j \leq 1$. Now $\operatorname{dim} U_{t}+\operatorname{dim} V_{t+1}=\operatorname{dim} N_{t}$ and $\operatorname{dim} U_{t+1}+$ $\operatorname{dim} V_{t+2}=\operatorname{dim} N_{t+1}$. By (a), $\operatorname{dim} V_{t+1} \leq \operatorname{dim} V_{t+2}$. Thus $\operatorname{dim} N_{t+1} \geq 2+$ $\operatorname{dim} N_{t}$. (2.1.1.A)

Case 2. Assume $C=L_{1}+L_{2}$ (union of two lines). There is an exact sequence

$$
0 \longrightarrow E_{L_{1}}(t) \longrightarrow E_{C}(t+1) \longrightarrow E_{L_{2}}(t+1) \longrightarrow 0 \text {. }
$$

In this case, it is possible $\delta^{-1}\left(N_{t+1}\right) \subseteq H^{0}\left(E_{L_{1}}(t+1)\right)$. So we can only 
conclude $\operatorname{dim} U_{t+1} \geq 1+\operatorname{dim} U_{t}$. Hence $\operatorname{dim} N_{t+1} \geq 1+\operatorname{dim} N_{t}$. The proof for the Case, $\Lambda^{2} E \cong \mathcal{O}_{Q_{2}}(-1)$, is similar and we will omit it.

(2) If $n_{-2}+3>n_{-1}$, then we can find an element $0 \neq x \in H^{0}\left(\mathcal{O}_{Q_{2}}(1)\right)$ such that $N_{-2} \stackrel{x}{\longrightarrow} N_{-1}$ is not injective. Let $C$ be the conic defined by $x$.

Case 1. Assume $C$ is nonsingular.

Then $\left.E\right|_{c} \cong \mathcal{O}_{P_{1} 1}(a) \oplus \mathcal{O}_{P^{1}}(-a)$ with $a \geq 2$, because $h^{0}\left(E_{C}(-1,-1)\right) \neq 0$. We consider the following diagram:

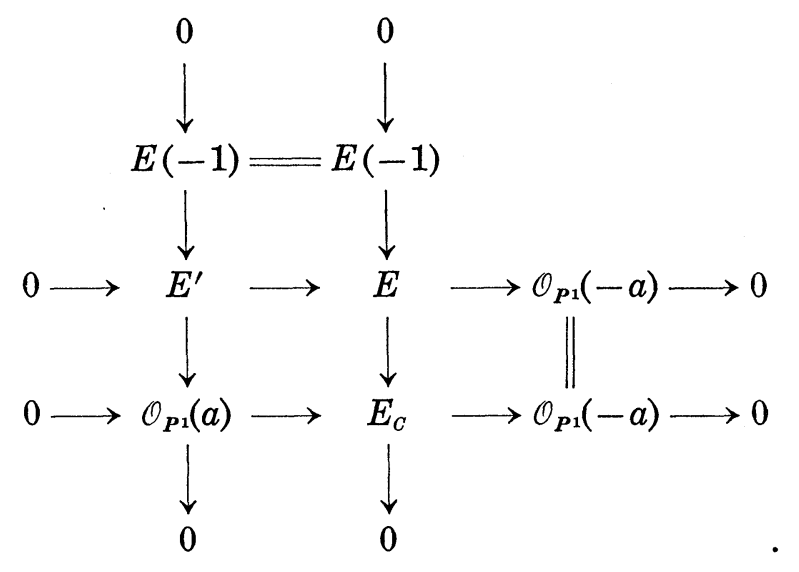

Since $E$ is stable, then $E^{\prime}$ is semistable and $h^{0}(E)=0$. Furthermore $\Lambda^{2} E^{\prime} \cong \mathcal{O}_{Q_{2}}(-1)$. There is the following exact sequence

$$
\underset{t}{\oplus} H^{0}\left(\mathcal{O}_{P^{1}}(a+2 t)\right) \stackrel{\delta}{\longrightarrow} \underset{t}{\oplus} H^{1}(E(-1+t)) \stackrel{\alpha}{\longrightarrow} \underset{t}{\oplus} H^{1}\left(E^{\prime}(t)\right) \text {. }
$$

Set $A=(N) \cap \operatorname{Im} \delta$ and $B=\operatorname{Im}\left(\left.\alpha\right|_{N}\right)$. Then $B$ is a submodule of $H^{1}\left(E^{\prime}(t)\right)$. There are the following exact sequences:

$$
0 \longrightarrow A_{-2} \longrightarrow N_{-2} \longrightarrow B_{-1} \longrightarrow 0
$$

and

$$
0 \longrightarrow A_{-1} \longrightarrow N_{-1} \longrightarrow B_{0} \longrightarrow 0 \text {. }
$$

$\operatorname{dim} N_{i}=\operatorname{dim} A_{i}+\operatorname{dim} B_{i+1}$ (for $i=-1$ and -2 ). Also $\operatorname{dim} A_{-1} \geq 2+$ $\operatorname{dim} A_{-2}$ and $\operatorname{dim} B_{0} \geq \operatorname{dim} B_{-1}$ (Lemma 2.1 (1) (a)). Thus $\operatorname{dim} N_{-1} \geq 2+$ $\operatorname{dim} N_{-2}$.

Case 2. Assume $C=L_{1}+L_{2}$.

Since $h^{0}\left(E_{c}(-1)\right) \neq 0$, either $\left.E\right|_{c} \cong \mathcal{O}_{c}(1) \oplus \mathcal{O}_{C}(-1)$ or we may assume $E_{L_{1}}=\mathcal{O}_{L_{1}}(a) \oplus \mathcal{O}_{L_{1}}(-a)$ with $a \geq 2$. In the case $E_{C} \cong \mathcal{O}_{C}(1) \oplus \mathcal{O}_{C}(-1)$, the 
argument given for the case that $C$ is nonsingular will also work here. So let us assume $E_{L_{1}}=\mathcal{O}_{L_{1}}(a) \oplus \mathcal{O}_{L_{1}}(-a)$ with $a \geq 2$. Without losing generality, we may assume $\mathcal{O}_{Q_{2}}\left(L_{1}\right) \cong \mathcal{O}_{Q_{2}}(1,0)$. We consider the following diagram:

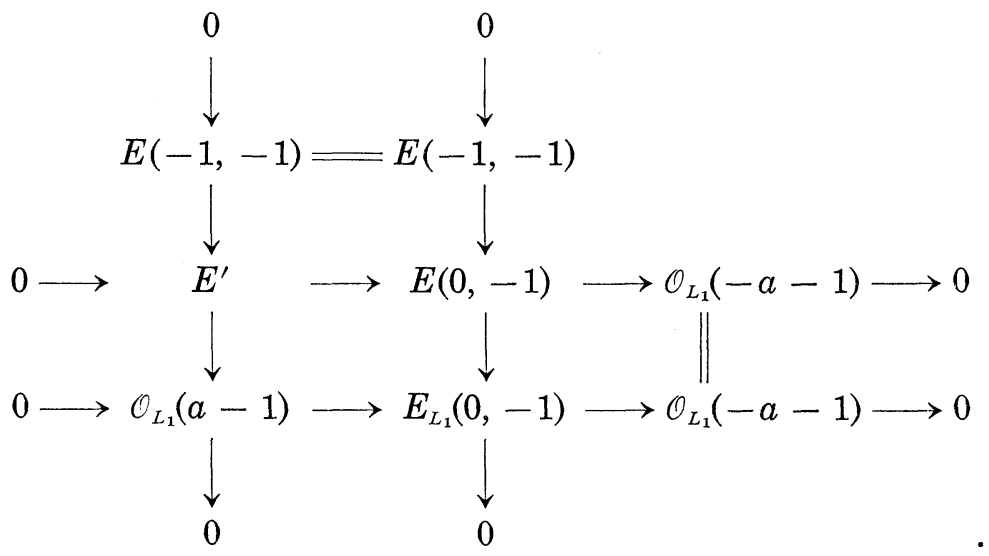

There is the exact sequence

$$
H^{0}\left(\mathcal{O}_{L_{1}}(a-1+t)\right) \stackrel{\delta^{\prime}}{\longrightarrow} \oplus_{t} H^{1}\left(E^{0}(-1+t)\right) \stackrel{\alpha^{\prime}}{\longrightarrow} H^{1}\left(E^{\prime}(t)\right) .
$$

Set $A^{\prime}=N \cap \operatorname{Im} \delta$ and $B^{\prime}=\left.\operatorname{Im} \alpha\right|_{N}$. Then

$$
\begin{aligned}
& 0 \longrightarrow A_{-2}^{\prime} \longrightarrow N_{-2} \longrightarrow B_{-1}^{\prime} \longrightarrow 0 \\
& 0 \longrightarrow A_{-1}^{\prime} \longrightarrow N_{-1} \longrightarrow B_{0}^{\prime} \longrightarrow 0 .
\end{aligned}
$$

Since $E_{H}^{\prime} \cong \mathcal{O}_{P_{1}}(-1) \oplus \mathcal{O}_{P_{1}}(-2)$ for a general hyperplane section $H$, $\operatorname{dim} B_{-1}^{\prime}$ $\leq \operatorname{dim} B_{0}^{\prime} . \quad$ Also $\operatorname{dim} A_{-1}^{\prime} \geq 1+\operatorname{dim} A_{0}^{\prime}$. Thus $\operatorname{dim} N_{-1} \geq 1+\operatorname{dim} N_{-2}$.

(3) Using the same notations as in the proof of 1 (b), we see $C=$ $L_{1}+L_{2}$ (2.1.1.A).

Also $\operatorname{dim} V_{t_{0}+2}=0$ (Lemma 2.1 (1) (b) and (2)). So $N_{j} \cong U_{j}$ for $j \leq$ $t_{0}+1$. Then the section $s \in H^{0}\left(\mathcal{O}_{Q_{2}}(1,0)\right)$ which define $L_{1}$ will have the desired property.

TheOREm 2.2. Let $E$ be a stable reflexive sheaf of rank 2 with $c_{1}(E)$ $=0$. (respectively, -1 ) on $Q_{3}$. Then there is a unique set of integers $\left\{k_{i}\right\}_{i=1,2, \cdots, c_{2}}$ (respectively, $\left\{k_{i}\right\}_{i=1,2, \cdots, c_{2}-1}$ ) called the spectrum of $E$ with the following properties: Let $\mathscr{H}=\oplus_{i} \mathcal{O}_{P^{1}}\left(k_{i}\right)$. Then

(1) (a) $h^{1}\left(Q_{3}, E(\ell)\right)=h^{0}(\mathscr{H}(\ell+1))$ for $\ell \leq-1$ (respectively, $\left.\ell \leq 0\right)$.

(b) $h^{2}(E(\ell))=h^{1}(\mathscr{H}(\ell+1))$ for $\ell \geq-2$. 
(2) (a) If an integer $k>0$ is in the spectrum, then $0,1, \cdots, k$ are also in the spectrum.

(b) If $k<0$ is in the spectrum, then $-1,-2, \cdots, k$ are also in the spectrum.

(3) $\sum k_{i}=\left(-c_{3}+c_{2}\right) / 2$ (respectively, $\left.-c_{3} / 2-c_{2}+1\right)$.

(4) If $E$ is a vector bundle, then $\left\{-k_{i}-1\right\}=\left\{k_{i}\right\}$

(respectively, $\left\{-k_{i}-2\right\}=\left\{k_{i}\right\}$ ).

Proof. (1) Let $Q_{2}$ be a general hyperplane section of $Q_{3}$ defined by $x \in H^{0}\left(\mathcal{O}_{Q_{3}}(1)\right.$ ), such that $E_{Q_{2}}$ is a stable vector bundle (Theorem 1.6). Let $M=\oplus_{j} H^{1}\left(E_{Q_{2}}(j)\right)$ and $N \subseteq M$ be the image of $\oplus_{j} H^{1}(E(j))$ under the restriction map. For $\ell \leq 0$, there is the following exact sequence,

$$
0 \longrightarrow H^{1}(E(\ell-1)) \longrightarrow H^{1}(E(\ell)) \longrightarrow N_{\ell} \longrightarrow 0 \text {. }
$$

So $\operatorname{dim} N_{\ell}=h^{1}(E(\ell))-h^{1}(E(\ell-1))$. We are looking for the set of integers $\left\{k_{i}\right\}$ satisfying (a) and (b). Condition (a) can be expressed as $h^{1}(E(\ell))=$ $\sum_{k \geq-\ell-1}\left(k_{i}+\ell+2\right)$. So $\operatorname{dim} N_{\ell}=\sum_{k_{i} \geq-\ell-1} 1=\sharp\left\{k_{i} \geq-\ell-1\right\}$. Since

$$
\operatorname{dim} N_{\ell}-\operatorname{dim} N_{\ell-1} \geq 0 \quad \text { for } \ell \leq-1
$$

(respectively, $\ell \leq 0$ ) by Lemma 2.1 , it is possible to find those integers $k_{i}$ satisfying condition (a). Furthermore those $k_{i} \geq 0$ (respectively $\geq-1$ ) are determined by (a). For (b), let $R$ be the kernel of

$$
\oplus_{j} H^{2}(E(j)) \stackrel{x}{\longrightarrow} \oplus_{j} H^{2}(E(j+1)) \text {. }
$$

For $\ell \geq-2$, there is the following exact sequence

$$
0 \longrightarrow R_{\ell+1} \longrightarrow H^{2}(E(\ell)) \longrightarrow H^{2}(E(\ell+1)) \longrightarrow 0 \text {. }
$$

So $\operatorname{dim} R_{\ell+1}=h^{2}(E(\ell))-h^{2}(E(\ell+1))$. Furthermore $R$ is a quotient module of $\oplus_{\ell} H^{1}\left(Q_{2}, E_{Q_{2}}(\ell)\right)$. Now condition (b) can be expressed as $h^{2}(E(\ell))=$ $\sum_{k_{i} \leq-\ell-3}-\left(k_{i}-\ell-2\right)$ for $\ell \geq-2 . \operatorname{dim} R_{\ell+1}=h^{2}(E(\ell))-h^{2}(E(\ell+1))=$ $\sum_{k_{i} \leq-\ell-3} 1=\sharp\left\{k_{i} \leq-\ell-3\right\}$. Since $\operatorname{dim} R_{\ell+1}-\operatorname{dim} R_{\ell+2} \geq 0$ by the dual formulation of Lemma 2.1 (a), we can find integers $\left\{k_{i}\right\}$ satisfying (b). Those integers $k_{i} \leq-1$ are uniquely determined by (b). If $c_{1}(E)=-1$, then we have two determination of $\sharp\left\{k_{i}\right\}=-1$, we have to check that they agree. (i.e., $\operatorname{dim} N_{0}-\operatorname{dim} N_{-1}=\operatorname{dim} R_{-1}-\operatorname{dim} R_{0}$ ). But this is clear, because $\operatorname{dim} N_{0}+\operatorname{dim} R_{0}=\operatorname{dim} N_{-1}+\operatorname{dim} R_{-1}=h^{1}\left(E_{Q_{2}}(-1)\right)=h^{1}\left(E_{Q_{2}}\right)$. Now $\operatorname{dim} N_{-1}+\operatorname{dim} R_{-1}=\sharp\left\{k_{i}\right\}=h^{1}\left(E_{Q_{2}}(-1)\right)=c_{2} \quad$ (respectively, $\left.c_{2}-1\right)$. So there are $c_{2}$ (respectively, $c_{2}-1$ ) integers in total. 
(2) This follows from the fact that if $N_{\ell} \neq 0$ and $\ell \leq-2$, then $\operatorname{dim} N_{\ell+1}-\operatorname{dim} N_{\ell}>0$ (Lemma 2.1 .1 (b) and (2)). Similarly by the dual formulation, if $\operatorname{dim} R_{\ell+1} \neq 0$, then $\operatorname{dim} R_{\ell}-\operatorname{dim} R_{\ell+1}>0$ for $\ell \geq-1$.

(3) Finally the proof for (3) and (4) are similar to the proof given in Propositions 7.2 and 7.3 in [7]. We shall omit it here.

THEOREM 2.3. Let $E$ be a stable rank 2 reflexive sheaf with $c_{1}(E)=0$ (respectively, $c_{1}(E)=-1$ ) on $Q_{3}$. Let $\left\{k_{i}\right\}_{i=1, \ldots, c_{2}}$ (respectively, $\left\{k_{i}\right\}_{i=1, \ldots, c_{2}-1}$ ) be its spectrum.

(a) If $k>1$ (respectively, $k \geq 1$ ) occurs in the spectrum, then $1, \cdots, k$ -1 occur at least twice (respectively, $0,1, \cdots, k-1$ ).

(b) If $k<-2$ occurs in the spectrum, then $k+1, \cdots,-2$ occur at least twice in the spectrum.

Proof. (a) Let $Q_{2}$ be a general hyperplane section of $Q_{3}$ defined by an element $\ell_{1} \in H^{0}\left(\mathcal{O}_{Q_{3}}(1)\right)$, such that $E_{Q_{2}}$ is a stable vector bundle. Let $N \subseteq \oplus_{j} H^{1}\left(E_{H}(j)\right)$ be the image of $\oplus_{j} H^{1}(E(j))$ under the restriction map. If for some $t(1 \leq t \leq k-1)$ occurs only once in the spectrum, then $\operatorname{dim} N_{-t-1}-\operatorname{dim} N_{-t-2}=1$ by the construction in Theorem 2.2. By Lemma 2.1 (3), there is an element $s \in H^{0}\left(\mathcal{O}_{Q_{2}}(1,0)\right)$ such that $x N_{m}=0$ for $m \leq$ $-t-1$ for any $x \in H^{0}\left(\mathcal{O}_{Q_{2}}(1,0)\right)$ where $s \mid x$. Let $\ell_{2} \in H^{0}\left(\mathcal{O}_{Q_{3}}(1)\right)$, such that $\ell_{2}$ is independent from $\ell_{1}$ and $\left.\ell_{2}\right|_{Q_{2}}$ is divisible by $s$. Let $m_{0}$ be the smallest integer such that $H^{1}\left(E\left(m_{0}\right)\right) \neq 0$. Consider $V=\operatorname{span}\left\{\ell_{1}, \ell_{2}\right\}$ and the multiplication map $\alpha: V \otimes H^{1}\left(E\left(m_{0}\right)\right) \rightarrow H^{1}\left(E\left(m_{0}+1\right)\right)$. Consider the following diagram:

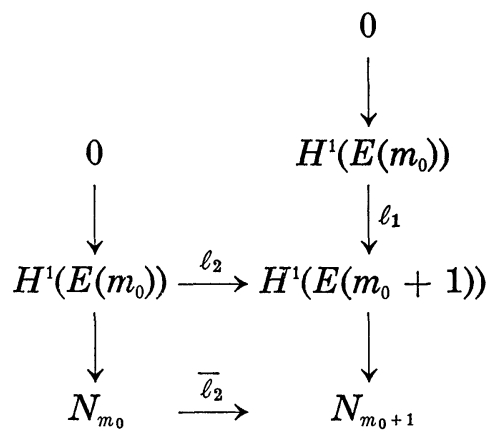

Since $\bar{\ell}_{2} \cdot N_{m_{0}}=0, \ell_{2}$ maps $H^{1}\left(E\left(m_{0}\right)\right)$ into the image of $\ell_{1}$. So $\operatorname{dim} \operatorname{Im} \alpha=$ $h^{1}\left(E\left(m_{0}\right)\right)$. By the bilinear map lemma, ([7], Lemma 5.1) there is an element $\ell_{2}^{\prime} \in V$, such that

$$
H^{1}\left(E\left(m_{0}\right)\right) \stackrel{\ell_{2}^{\prime}}{\longrightarrow} H^{1}\left(E\left(m_{0}+1\right)\right)
$$


is not injective. Let $Q_{2}^{\prime}$ be another general hyperplane section of $Q_{3}$, such that $E_{Q_{2}^{\prime}}$ is stable and $\ell_{2}^{\prime} \mid Q_{2}^{\prime}$ defined a nonsingular conic. By the same argument, there is an element $s^{\prime} \in H^{0}\left(\mathcal{O}_{Q_{2}^{\prime}}(1,0)\right)$ such that if $x \in H^{0}\left(\mathcal{O}_{Q_{2}^{\prime}}(1)\right)$ is divisible by $s$, then $x \cdot N_{m}^{\prime}=0$ for $m \leq-t-1$, where $N^{\prime}$ is the image of $\oplus_{j} H^{1}(E(j))$ in $\oplus_{j} H^{1}\left(E_{Q_{2}}(j)\right)$. Consider the following diagram:

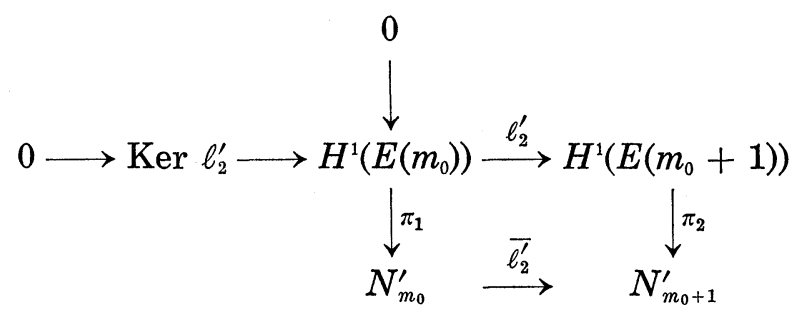

Since $\ell_{2}^{\prime}$ defines a nonsingular conic, $\ell_{2}^{\prime}$ is not divisible by $s^{\prime}$ and $\overline{\ell_{2}^{\prime}}: N_{m_{0}}^{\prime}$ $\rightarrow N_{m_{0}+1}^{\prime}$ is injective (Lemma 2.1 (3)). Now $\overline{\ell_{2}^{\prime}} \circ \pi_{1}$ is injective but $\pi_{2} \ell_{2}^{\prime}$ is not injective, which is a contradiction.

Corollary 2.4. Let $E$ be a stable rank 2 vector bundle on $Q_{3}$ with $c_{1}(E)=0$ (respectively, $c_{1}(E)=-1$ ). Then $h^{1}(E(\ell))=0$ for $\ell \leq\left(-c_{2} / 4\right)-1$ (respectively, $-c_{2} / 4$ ).

Proof. Using Theorem 2.2 and Theorem 2.3, we see it will be sufficient to show $S=\{-k-3,-k-2,-k-2,-k-1,-k-1, \cdots,-2,-2$, $-1,0,1,1, \cdots, k, k, k+1\}$ is not spectrum of a stable rank 2 vector bundle on $Q_{3}$. Suppose for contradiction that $S$ is the spectrum of $E$. Then $c_{2}(E)=4 k+4$. Also $h^{1}(E(-k-2))=1$ and $h^{1}(E(-k-1))=4$. So the $\operatorname{map} H^{0}\left(\mathcal{O}_{Q_{3}}(1)\right) \otimes H^{1}(E(-k-2)) \rightarrow H^{1}(E(-k-1))$ is not injective. Let $x \in$ $H^{0}\left(\mathcal{O}_{Q_{3}}(1)\right)$ such that

$$
H^{1}(E(-k-2)) \stackrel{x}{\longrightarrow} H^{1}(E(-k-1))
$$

is not injective. Let $Q_{2}$ be the corresponding hyperplane. Since

$$
h^{0}\left(E_{Q_{2}}(-k-1)\right) \neq 0, \quad h^{0}\left(E_{Q_{2}}\right) \geq 1+(2 k+2)+(k+1)^{2} .
$$

On the other hand, $h^{1}(E(-1))=(2 k+2)+(k+1)^{2}$ from the spectrum. By considering the exact sequence $H^{0}(E) \rightarrow H^{0}\left(E_{Q_{2}}\right) \rightarrow H^{1}(E(-1))$, we see $h^{0}(E) \neq 0$ which is a contradiction.

Remark. The following example will show the bound given in Corollary 2.4 is the best possible. Let $Z$ be two disjoint conic in $Q_{3}$. Using $Z$ we can construct the following extension, 


$$
0 \longrightarrow \mathcal{O}_{Q_{3}} \longrightarrow F(1) \longrightarrow I_{Z}(2) \longrightarrow 0
$$

where $c_{1}(F)=0$ and $c_{2}(F)=2$. Let $Y=Y_{1} \amalg Y_{2}$ where $Y_{1}$ is the zero set of a general section of $F(m)$ and $Y_{2}$ is a curve of the type $(2 m-1,2 m-1)$ on a nonsingular quadric. Using $Y$ we can construct the following extension:

$$
0 \longrightarrow \mathcal{O} \longrightarrow E(m) \longrightarrow I_{Y}(2 m) \longrightarrow 0
$$

where $E$ is a rank 2 bundle with $c_{1}(E)=0$ and $c_{2}(E)=4 m$. Also $h^{1}(E(-m))=h^{1}\left(I_{Y}\right)=1$.

\section{REFERENCES}

[1] Barth, W., Moduli of vector bundles on the projective plane, Invent. Math., 42 (1977), 63-91.

[2] - Some properties of stable vector bundles on $\boldsymbol{P}^{n}$, Math. Ann., 226 (1977), 125-150.

[ 3 ] Barth, W., Elencwajg, G., Concernant la cohomologie des fibres algebriques stable sur $P n(C)$, In: Varietes analytiques compacts (Nice, 1977). Lecture Notes in Math., 683 (1978), 1-24.

[ 4 ] Ein, L., Stable vector bundles in char p>0, Math. Ann., 254 (1980), 53-72.

[ 5 ] Ein, L., Hartshorne, R., Vogelaur, H., Restriction theorems for stable rank 3 vector bundles $\boldsymbol{P}^{n}$, Math. Ann., 259 (1982), 541-569.

[6] Elencwajg, G., Hirschowitz, A. and Schneider, M., Type de scindage generalise pour fibres stables, (Preprint).

[ 7 ] Hartshorne, R., Stable reflexive sheaves, Math. Ann., 254 (1980), 121-176.

[ 8 ] — Stable reflexive sheaves II, Invent. Math., 66 (1982), 165-190.

[ 9 ] Hartshorne, R., Sols, I., Stable rank 2 vector bundle on $\boldsymbol{P}^{3}$ with $c_{1}=-1, c_{2}=2$, Crelle J., 325 (1981), 145-152.

Department of Mathematics

Massachusetts Institute of Technology

Cambridge, Mass. 02139

USA 